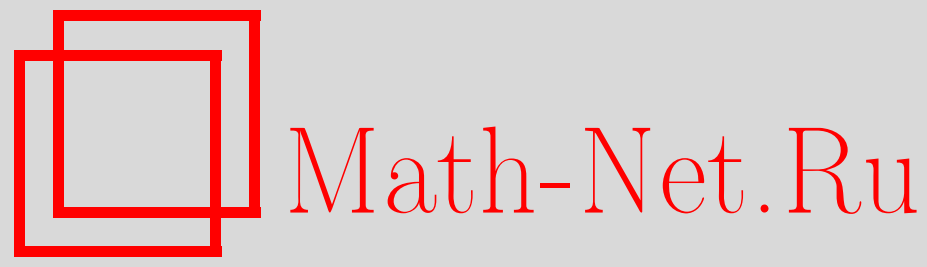

А. А. Доси, Теорема о биполяре для квантовых конусов, Функи. анализ и его прил., 2012, том 46, выпуск $3,84-89$

DOI: https://doi.org/10.4213/faa3084

Использование Общероссийского математического портала Math$\mathrm{Net.Ru} \mathrm{подразумевает,} \mathrm{что} \mathrm{вы} \mathrm{прочитали} \mathrm{и} \mathrm{согласны} \mathrm{с} \mathrm{пользователь-}$ ским соглашением

http://www . mathnet.ru/rus/agreement

Параметры загрузки:

IP: 34.239 .49 .27

26 апреля 2023 г., 11:48:06

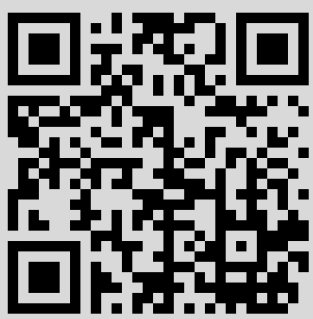


$\operatorname{codim} M \leqslant s$ и существует $y \in \mathbb{R}^{k}, y \geqslant 0, y \neq 0$,

такой, что $y Q(x) \geqslant 0$ для любого $x \in M$

соответственно.

Из предложения 3 и того, что если выполняется (4), то условия (1) и (b) эквивалентны, получаем следующее утверждение:

Условие (2) эквивалентно тому, что $\bigcup_{M \in \mathscr{M}_{k-2}^{++}} M=X$.

Применяя это утверждение к возмущенным квадратичным формам $q_{i, \varepsilon}(x)=$ $q_{i}(x)+\varepsilon|x|^{2}$, где $\varepsilon>0$ - параметр возмущения, и осуществляя предельный переход при $\varepsilon \rightarrow 0$, с помощью теоремы 4.4 из [3, с. 29] получаем такое утверждение:

Условие (3) эквивалентно тому, что $\bigcup_{M \in \mathscr{M}_{k-2}^{+}} M=X$.

Я приношу искреннюю благодарность профессору Р. С. Исмагилову за плодотворные обсуждения и ценные предложения по этой работе.

\title{
ЛитЕРАТУРА
}

[1] J.-B. Hiriart-Urruty, SIAM Review, 49:2 (2007), 255-273. [2] А. В. Арутюнов, Матем. заметки, 84:2 (2008), 163-174. [3] А. В. Арутюнов, Условия экстремума. Анормальные и вырожденные задачи, Факториал, М., 1997.

Российский университет дружбы народов e-mail: arutun@orc.ru

Поступило в редакцию 28 сентября 2010 г.

УДК 517.98

\section{Теорема о биполяре для квантовых конусов}

\author{
(c) 2012. А. Доси
}

Одним из фундаментальных результатов квантового функционального анализа является операторная (или квантовая) теорема о биполяре, доказанная Эффросом и Уэбстером в [5], в которой утверждается, что двойная квантовая поляра для абсолютно матрично выпуклого множества на линейном пространстве $V$ совпадает со своим слабым замыканием относительно фиксированной дуальной пары $(V, W)$. Известная теорема Руана $([7,2.3 .5],[10])$ об абстрактной характеризации операторных (или квантовых нормированных) пространств легко выводится из операторной теоремы о биполяре. С другой стороны, как показано в [6], результат Руана вместе с некоторыми фактами из теории двойственности для операторных пространств позволяет получить другое доказательство операторной теоремы о биполяре. Это ее доказательство на языке операторных пространств. Таким образом, и теорема Руана, и операторная теорема о биполяре имеют единый источник (в теории $C^{*}$-алгебр); в определенном смысле они эквивалентны. Другой подход к теореме Руана был предложен Полсеном в [13]. Это иная точка зрения на предмет, использующая структуры *-линейного пространства и матричного порядка, которая приводит к концепции операторной системы. Каждое абстрактное операторное пространство вложено в абстрактную операторную систему с точностью до матричной изометрии. Эта 
система, в свою очередь, вложена в $\mathscr{B}(H)$ с точностью до изоморфизма относительно матричного порядка; этот результат об абстрактной характеризации операторных систем принадлежит Чои и Эффросу [1].

Локально выпуклая версия операторной системы в ее конкретной форме введена в [2]. Такие системы определяются как унитальные эрмитовы подпространства мультинормированной $C^{*}$-алгебры $C_{\mathscr{E}}^{*}(\mathscr{D})$ всех некоммутативных непрерывных функций на квантовой области $\mathscr{E}$. Унитальное самосопряженное подпространство $V \subseteq C_{\mathscr{E}}^{*}(\mathscr{D})$ называется квантовой (или локально операторной) системой. Приложения к квантовым проблемам моментов рассмотрены в [3], [4]. Ключевую роль в теории квантовых систем играет понятие локально матричной положительности. С помощью этого понятия локально выпуклая версия теоремы об инъективности Чои-Эффроса [1] доказана в [2] для квантовых систем. Таким образом, «конкретная» локально матричная положительность корректно определена и играет существенную роль в различных нетривиальных результатах о квантовых системах. Поэтому нам нужно выяснить, что стоит за этой конкретной локально матричной положительностью, а именно, как это понятие может быть описано в абстрактной форме.

В данной заметке мы исследуем свойства двойственности квантовых конусов как подходящий язык, чтобы обрисовать локально матричную положительность в общей форме. Двойственность квантовых конусов представляет самостоятельный интерес и оказывается одним из фундаментальных разделов теории квантовых пространств. В качестве главного результата мы предлагаем теорему о биполяре для квантовых конусов. Теорема о биполяре для квантовых конусов основана на классической теореме о строгой отделимости $[11,10.4 .8]$ и представляется простой и естественной. Привлекая конструкцию Полсена $\mathscr{P}_{V}$, мы можем вывести операторную теорему о биполяре из теоремы о биполяре для квантовых конусов. Это новое прямое доказательство операторной теоремы о биполяре (в частности, теоремы Руана), использующее методы самой теории квантовых пространств.

1. Квантовые пространства. Линейное пространство всех матриц конечного размера $x=\left[x_{i j}\right]$ над линейным пространством $V$ обозначается через $M(V)$, и мы полагаем $M=M(\mathbb{C})$. Это пространство $M$ является нормированным пространством, отождествляемым с пространством операторов конечного ранга на гильбертовом пространстве $\ell_{2}$, пополнением которого является $C^{*}$-алгебра компактных операторов $[12,6.3 .2]$. Если $v, w \in M(V)$, то имеется прямая сумма $v \oplus w \in M(V)$. Если $a \in M, v \in M(V)$ и $b \in M$ с согласованными размерами, то имеется матричное произведение avb $=\left[\sum_{k, l} a_{i k} v_{k l} b_{l j}\right]_{i, j} \in M(V)$. Линейное отображение $\varphi: V \rightarrow W$ имеет каноническое линейное расширение $\varphi^{(\infty)}: M(V) \rightarrow M(W), \varphi\left(\left[x_{i j}\right]\right)=\left[\varphi\left(x_{i j}\right)\right]$. Под квантовым множеством $\mathfrak{B}$ на $V$ мы понимаем семейство $\mathfrak{B}=\left(\mathfrak{b}_{n}\right)$ подмножеств $\mathfrak{b}_{n} \subseteq M_{n}(V)\left(M_{n}(V)-\right.$ пространство $(n \times n)$-матриц над $V), n \geqslant 1$. Квантовое множество $\mathfrak{B}$ в $M(V)$ называется матрично выпуклым [9], если $\mathfrak{B} \oplus \mathfrak{B} \subseteq \mathfrak{B}$ и $a^{*} \mathfrak{B} a \subseteq \mathfrak{B}, a \in M$, $a^{*} a=I$. Матрично выпуклое множество называется абсолютно матрично вы-

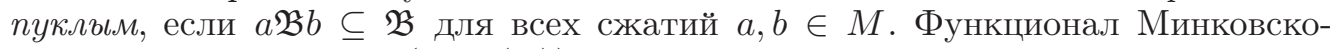
го для поглощающего (в $M(V)$ ) абсолютно матрично выпуклого множества называется матричной полунормой на $V$. Полинормированная (или локально выпуклая) топология, определенная отделяющим семейством матричных полунорм, называется квантовой топологией, а линейное пространство $V$, наделенное квантовой топологией, называется квантовым пространством. Квантовое 
пространство, в котором квантовая топология определена матричной нормой, называется квантовым нормированным (или абстрактным операторным) пространством (см. [10]). Подпространства $C^{*}$-алгебры $\mathscr{B}(H)$ всех ограниченных линейных операторов на гильбертовом пространстве $H$ являются (конкретными) операторными пространствами. Таким образом, квантовая топология $\mathfrak{t}$ на $V$ - это полинормированная топология в $M(V)$ с базой фильтра окрестностей, состоящей из абсолютно матрично выпуклых множеств. В частности, она индуцирует полинормированную топологию $\mathfrak{t} \mid V$ в $V$. Обратно, каждая полинормированная топология $t$ в $V$ является сужением некоторой квантовой топологии $\mathfrak{t}$ в $M(V)$, называемой ее квантованием, т.е. $t=\mathfrak{t} \mid V$. Все эти квантования оцениваются квантованиями $\min t$ и $\max t[5]$. Пусть $(V, W)$ является дуальной парой с двойственностью $\langle\cdot, \cdot\rangle: V \times W \rightarrow \mathbb{C}$. Данное спаривание определяет квантовое (или матричное) спаривание $\langle\langle\cdot, \cdot\rangle\rangle: M(V) \times M(W) \rightarrow M$, $\langle\langle v, w\rangle\rangle=\left[\left\langle v_{i j}, w_{s t}\right\rangle\right]_{(i, s),(j, t)}$. Семейство $\left\{p_{w}: w \in M(W)\right\}$ с $p_{w}(v)=\|\langle\langle v, w\rangle\rangle\|$ матричных полунорм определяет слабую квантовую топологию $\mathfrak{s}(V, W)$ на $V$, которая является единственным квантованием слабой топологии $\sigma(V, W)$ (см. [6]). Итак, $\min \sigma(V, W)=\max \sigma(V, W)=\mathfrak{s}(V, W)$. Для данного квантового множества $\mathfrak{B}$ в $M(V)$ определим его абсолютную матричную поляру $\mathfrak{B}^{\odot}=\{w \in M(W): \sup \|\langle\langle\mathfrak{B}, w\rangle\rangle\| \leqslant 1\}$ в $M(W)$. Легко проверить, что $\mathfrak{B}^{\odot}$ является $\mathfrak{s}(W, V)$-замкнутым абсолютно матрично выпуклым множеством в $M(W)$ (см. [5]). Линейное отображение $\varphi: V \rightarrow V^{\prime}$ между квантовыми пространствами называется матрично непреръвным, если $\varphi^{(\infty)}: M(V) \rightarrow M\left(V^{\prime}\right)$ является непрерывным линейным отображением соответствующих полинормированных пространств. Для квантовых нормированных пространств мы имеем дело с матричным ограниченным линейным отображением. Напомним, что линейное отображение $\varphi: V \rightarrow V^{\prime}$ квантовых нормированных пространств называется матричной изометрией, если $\left\|\varphi^{(\infty)}(v)\right\|_{M\left(V^{\prime}\right)}=\|v\|_{M(V)}$ для всех $v \in M(V)$.

Под инволющией на линейном пространстве $X$ мы понимаем линейное отображение сопряжения $x \mapsto x^{*}$ на $X$, такое, что $x^{* *}=x$ для всех $x \in X$. Линейное пространство, наделенное инволюцией, называется *-линейным пространством. Элемент $x \in X$ называется эрмитовым, если $x^{*}=x$. Множество всех эрмитовых элементов обозначается через $X_{h}$, оно является вещественным линейным подпространством в $X$. Инволюция на $X$ естественным образом расширяется до инволюции на матричном пространстве $M(X)$. Таким образом, $M(X)$ превращается в $*$-линейное пространство. Если $X$ является $*$-линейным пространством и $(X, Y)$ - такая дуальная пара, что инволюция на $X$ является $\sigma(X, Y)$-непрерывной, то $Y$ обладает канонической инволюцией $y \mapsto y^{*}$, $\left\langle x, y^{*}\right\rangle=\left\langle x^{*}, y\right\rangle^{*}$. В этом случае будем говорить, что $(X, Y)$ является дуальной *-nарой.

Пусть $(J, n)$ - упорядоченная пара, состоящая из множества $J$ и фиксированного отображения $n: J \rightarrow \mathbb{N}$. Для каждого $w \in J$ существует атомная алгебра $M_{n(w)}$ всех квадратных комплексных матриц порядка $n(w)$. В частности, имеется $L^{\infty}$-алгебра фон Неймана $M_{(J, n)}=\bigoplus_{w \in J}^{\infty} M_{n(w)}$, которая является прямой $L^{\infty}$-суммой атомных алгебр. Отметим, что $M_{(J, n)}$ является унитальной *-подалгеброй в прямом произведении $D_{(J, n)}=\prod_{w \in J} M_{n(w)}$ атомных алгебр. Поэтому каждый элемент $v \in M_{(J, n)}$ можно записать как семейство $v=\left(v_{w}\right)_{w \in I}$ с $v_{w} \in M_{n(w)}$ и $\|v\|=\sup _{w \in J}\left\|v_{w}\right\|<\infty$. 
2. Квантовые конусы. Фиксируем *-линейное пространство $X$. Квантовое подмножество $\mathfrak{C}$ в $M(X)_{h}$ называется квантовым конусом на $X$, если $\mathfrak{C}+\mathfrak{C} \subseteq \mathfrak{C}$ и $a^{*} \mathfrak{C} a \subseteq \mathfrak{C}$ для всех $a \in M$. Квантовый конус $\mathfrak{C}$ называется квантовым *-конусом, если $M(X)_{h}=\mathfrak{C}-\mathfrak{C}$. Квантовый конус $\mathfrak{C}$ на $X$ называется отделимымм квантовым конусом на $X$, если $\mathfrak{C} \cap-\mathfrak{C}=\{0\}$. Любая $C^{*}$-алгебра $A$ обладает отделимым квантовым *-конусом $M(A)_{+}$, совпадающим с множеством всех положительных элементов в $M(A)$. Далее, пусть $(J, n)$ - упорядоченная пара, где $n$ - отображение $J \rightarrow \mathbb{N}$. Если $K$ - подмножество в $J$, то мы имеем квантовый $*$-конус $\mathfrak{C}_{K}=\left\{x \in M\left(D_{(J, n)}\right)_{h}: x_{w} \geqslant 0, w \in K\right\}$ на $D_{(J, n)}$. Отметим, что $\mathfrak{C}_{J}=M\left(D_{(J, n)}\right)+$ является отделимым квантовым $*$-конусом.

Пусть теперь $V$ - произвольное линейное пространство. Если $V^{*}-$ сопряженное к нему пространство, то введем следующее *-линейное пространство: $\mathscr{P}_{V}=\left\{\left[\begin{array}{cc}\lambda & u \\ v^{*} & \mu\end{array}\right]: \lambda, \mu \in \mathbb{C}, u, v \in V\right\}$ с инволюцией $\left[\begin{array}{cc}\lambda & u \\ v^{*} & \mu\end{array}\right]^{*}=\left[\begin{array}{cc}\lambda^{*} & v \\ u^{*} & \mu^{*}\end{array}\right]$. Отметим, что $\mathscr{P}_{V}=\mathbb{C} \oplus \mathbb{C} \oplus V \oplus V^{*}$ является линейным пространством. С точностью до канонического перемешивания мы имеем $M\left(\mathscr{P}_{V}\right)=\left\{\left[\begin{array}{cc}a & x \\ y^{*} & b\end{array}\right]: a, b \in M\right.$, $x, y \in M(V)\}$, где $y^{*}=\left[y_{j i}^{*}\right] \in M\left(V^{*}\right)$, тогда как $y=\left[y_{i j}\right] \in M(V)$. Если $(V, W)$ является дуальной парой, то дуальной парой является и $\left(\mathscr{P}_{V}, \mathscr{P}_{W}\right)$ с канонической двойственностью $\mathscr{P}_{V} \times \mathscr{P}_{W} \rightarrow \mathbb{C},\left\langle\left[\begin{array}{cc}\lambda & u \\ v^{*} & \mu\end{array}\right],\left[\begin{array}{cc}\alpha & z \\ w^{*} & \beta\end{array}\right]\right\rangle=\lambda \alpha^{*}+$ $\mu \beta^{*}+\langle u, z\rangle+\langle v, w\rangle^{*}$, где $u, v \in V$ и $z, w \in W$. Само пространство $V$ вложено в $\mathscr{P}_{V}$ посредством канонического линейного отображения $\iota_{V}: V \rightarrow \mathscr{P}_{V}$, $\iota_{V}(x)=\left[\begin{array}{ll}0 & x \\ 0 & 0\end{array}\right]$. Если $\mathfrak{B} \subseteq M(V)$ - квантовое множество, то определим новое квантовое множество $\mathfrak{C}_{\mathfrak{B}}$ на пространстве $\mathscr{P}_{V}, \mathfrak{C}_{\mathfrak{B}}=\left\{\left[\begin{array}{cc}a & v \\ v^{*} & b\end{array}\right] \in M\left(\mathscr{P}_{V}\right)_{h}:\right.$ $\left.(a+\varepsilon I)^{-1 / 2} v(b+\varepsilon I)^{-1 / 2} \in \mathfrak{B}, a, b \in M_{+}, \varepsilon>0, v \in M(V)\right\}$. Отметим, что $\mathfrak{C}_{\mathfrak{B}}$ является квантовым (*-)конусом на $\mathscr{P}_{V}$, если $\mathfrak{B}-$ (поглощающее) абсолютно матрично выпуклое множество.

Лемма 1. Пусть $(V, W)$ - дуальная пара, и пусть $\mathfrak{B}$ - абсолютно матрично выпуклое множество на $V$. Тогда $\mathfrak{C}_{\mathfrak{B}-}=\mathfrak{C}_{\mathfrak{B}}^{-}$, где $\mathfrak{C}_{\mathfrak{B}}^{-}$есть $\mathfrak{s}\left(\mathscr{P}_{V}, \mathscr{P}_{W}\right)$ замъкание квантового множества $\mathfrak{C}_{\mathfrak{B}}$ и $\mathfrak{B}^{-}$есть $\mathfrak{s}(V, W)$-замъкание квантового множества $\mathfrak{B}$. В частности, $\mathfrak{C}_{\mathfrak{B}}$ является отделимым квантовым конусом, если и только если им является $\mathfrak{B}$ в том смысле, что $\bigcap_{\varepsilon>0} \varepsilon \mathfrak{B}=\{0\}$.

3. Квантовая поляра. Пусть теперь $(X, Y)$ - дуальная *-пара. Если $\mathfrak{C}$ является квантовым множеством на $X$, то определим его квантовую поляру $\mathfrak{C}^{\square}=\left\{y \in M(Y)_{h}:\langle\langle\mathfrak{C}, y\rangle\rangle \geqslant 0\right\}$ в $M(Y)$. Легко проверить, что $\mathfrak{C}^{\square}$ является квантовым конусом в $M(Y)$.

Предложение 2. Пусть $(V, W)$ - дуальная пара, и пусть $\mathfrak{B}$ является абсолютно матрично выпуклым множеством на $V$. Тогда $\mathfrak{C}_{\mathfrak{B}}^{\bullet}=\mathfrak{C}_{\mathfrak{B} \odot}$.

Теперь приведем теорему о биполяре для слабо замкнутого квантового *-конуса.

Теорема 3. Пусть $(X, Y)$ - дуальная *-пара, и пусть $\mathfrak{C}$ является слабо замкнутым квантовъмм *-конусом на $X$. Тогда $\mathfrak{C}=\mathfrak{C}^{\square}$. 
Следствие 4. Пусть $(V, W)$ - дуальная пара, и пусть $\mathfrak{B}$ является абсолютно матрично выпуклым множеством на $X$. Тогда $\mathfrak{B}^{-}=\mathfrak{B}^{\odot \odot}$. $\left(\mathfrak{B}^{-}\right.$есть $\mathfrak{s}(V, W)$-замыкание квантового множества $\mathfrak{B}$.)

Доказательство. Можно предположить, что $\mathfrak{B}$ является слабо замкнутым поглощающим и абсолютно матрично выпуклым множеством. Рассмотрим дуальную $*$-пару $\left(\mathscr{P}_{V}, \mathscr{P}_{W}\right)$. По лемме 1 квантовый $*$-конус $\mathfrak{C}_{\mathfrak{B}}$ является $\mathfrak{s}\left(\mathscr{P}_{V}, \mathscr{P}_{W}\right)$-замкнутым. Более того, $\mathfrak{C}_{\mathfrak{B}}^{\bullet}=\mathfrak{C}_{\mathfrak{B} \odot}$ благодаря предложению 2. Так как $\mathfrak{B} \odot$ является $\mathfrak{s}(W, V)$-замкнутым квантовым множеством на $W$, то $\mathfrak{C}_{\mathfrak{B} \odot}^{\bullet}=$ $\mathfrak{C}_{\mathfrak{B} \odot \odot}$ снова благодаря предложению 2. Используя теорему 3 (примененную к

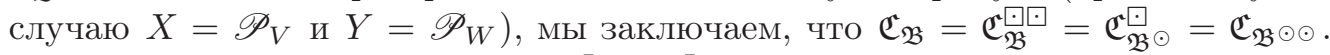
Наконец, пусть $v \in \mathfrak{B} \odot \odot$. Тогда $\left[\begin{array}{cc}I & v \\ v^{*} & I\end{array}\right] \in \mathfrak{C}_{\mathfrak{B} \odot \odot}=\mathfrak{C}_{\mathfrak{B}}$. Отсюда следует, что $(1+\varepsilon)^{-1} v \in \mathfrak{B}$ для всех $\varepsilon>0$. Но $v=\mathfrak{s}(V, W)-\lim _{\varepsilon}(1+\varepsilon)^{-1} v$, т. е. $v \in \mathfrak{B}^{-}=\mathfrak{B}$. Поэтому $\mathfrak{B}=\mathfrak{B} \odot \odot$, что доказывает утверждение.

Теперь приведем теорему о представлении для квантового конуса.

Следствие 5. Пусть $(X, Y)$ - дуальная *-пара, и пусть $\mathfrak{C}-$ слабо замкнутый квантовый *-конус на $X$. Тогда существуют упорядоченная пара $(J, n)$ u *-линейное отображение $\varphi: X \rightarrow D_{(J, n)}$, такие, что $\varphi^{(\infty)}(\mathfrak{C})=\varphi^{(\infty)}\left(M(X)_{h}\right) \cap$ $M\left(D_{(J, n)}\right)_{+} \cdot$

Доказательство. Пусть $J=\mathfrak{C}^{\square}$, причем $n: \mathfrak{C}^{\square} \rightarrow \mathbb{N}, n(y)=m$, где $y \in$ $M_{m}(Y)$. Мы имеем корректно определенное $*$-линейное отображение $\varphi: X \rightarrow$ $D_{(J, n)}, \varphi(x)=\left(\langle\langle x, y\rangle)_{y \in J}\right.$. Легко проверить, что $\varphi^{(\infty)}(x)=(\langle\langle x, y\rangle\rangle)_{y \in J}, x \in$ $M(X)$. Если $x \in \mathfrak{C}$, то $\langle\langle x, y\rangle\rangle \geqslant 0, y \in J$, при $J=\mathfrak{C}^{\square}$. Таким образом, $\varphi^{(\infty)}(x) \in$ $M\left(D_{(J, n)}\right)_{+}$. Обратно, если $\varphi^{(\infty)}(x) \in M\left(D_{(J, n)}\right)_{+}$для некоторого элемента $x \in$ $M(X)_{h}$, то $\langle\langle x, y\rangle\rangle \geqslant 0$ для всех $y \in \mathfrak{C}^{\square}$. Это означает, что $x \in \mathfrak{C}^{\square}$. По теореме 3 $x \in \mathfrak{C}$. Таким образом, $\varphi^{(\infty)}(\mathfrak{C})=\varphi^{(\infty)}\left(M(X)_{h}\right) \cap M\left(D_{(J, n)}\right)_{+}$.

Следствие 6 (теорема Руана). Пусть $V-$ квантовое нормированное пространство. Тогда $V \subseteq M_{(J, n)}$ с точностью до матричной изометрии для некоторой упорядоченной пары $(J, n)$.

Доказательство. Рассмотрим единичный шар ball $M(V)$ в $M(V)$ и возьмем его абсолютную матричную поляру $\mathfrak{B}=(\text { ball } M(V))^{\odot}$ в $M\left(V^{*}\right)$, где $V^{*}$ - пространство всех ограниченных линейных функционалов на $V$. Мы имеем корректно определенное отображение $n: \mathfrak{B} \rightarrow \mathbb{N}, n(w)=n$, если $w \in \mathfrak{b}_{n}$. Поэтому $(\mathfrak{B}, n)$ является упорядоченной парой. Более того, мы имеем корректно определенное сжатие $\Phi: V \rightarrow M_{(\mathfrak{B}, n)}, \Phi(x)=\bigoplus_{w \in \mathfrak{B}}\langle\langle x, w\rangle\rangle$. Если $v \in$ ball $M(V)$, то $\Phi^{(\infty)}(v)=\left[\Phi\left(v_{i j}\right)\right]=\left[\bigoplus_{w \in \mathfrak{B}}\left\langle\left\langle v_{i j}, w\right\rangle\right\rangle\right]=\bigoplus_{w \in \mathfrak{B}}\langle\langle v, w\rangle\rangle$ и $\left\|\Phi^{(\infty)}(v)\right\|=\sup \|\langle\langle v, \mathfrak{B}\rangle\rangle\|$ $\leqslant 1$ для $\mathfrak{B}=(\text { ball } M(V))^{\odot}$. Поэтому $\Phi$ является матричным сжатием. Если $\Phi$ не является матричной изометрией, то существует такой элемент $v \in M(V)$, $\|v\|=1$, что $\left\|\Phi^{(\infty)}(v)\right\| \leqslant \alpha<1$. Отсюда следует, что $\sup _{u \in \alpha^{-1} \mathfrak{B}}\|\langle\langle v, u\rangle\rangle\| \leqslant 1$, т. е. $v \in\left(\alpha^{-1} \mathfrak{B}\right)^{\odot}$. Но $\left(\alpha^{-1} \mathfrak{B}\right)^{\odot}=\alpha \mathfrak{B}^{\odot}$ и ball $M(V)$ является $\mathfrak{s}\left(V, V^{*}\right)$-замкнутым. Мы заключаем, что ball $M(V)=\mathfrak{B}^{\odot}$ в силу следствия 4 . Значит, $v \in \alpha$ ball $M(V)$ или $\|v\| \leqslant \alpha<1$; возникает противоречие. Таким образом, $V \subseteq M_{(\mathfrak{B}, n)}$ с точностью до матричной изометрии. 


\section{ЛИТЕРАТУРА}

[1] M. D. Choi, E. G. Effros, J. Funct. Anal., 24:2 (1977), 156-209. [2] A. A. Dosiev, J. Funct. Anal., 255:7 (2008), 1724-1760. [3] A. A. Dosiev, C. R. Math. Acad. Sci. Paris, 344:10 (2007), 627-630. [4] A. A. Dosi, Trans. Amer. Math. Soc., 363:2 (2011), 801856. [5] A. A. Dosi, J. Math. Phys., 51:6 (2010), 1-43. [6] A. A. Dosi, Inter. J. Math., 22:4 (2011), 1-7. [7] E. G. Effros, Z-.J. Ruan, Operator Spaces, Clarendon Press, Oxford, 2000. [8] E. G. Effros, C. Webster, in: Operator Algebras and Applications (Samos 1996), NATO Adv. Sci. Inst. Ser. C Math. Phys. Sci., vol. 495, Kluwer Acad. Publ., Dordrecht, 1997, 163-207. [9] E. G. Effros, S. Winkler, J. Funct. Anal., 144:1 (1997), 117-152. [10] А. Я. Хелемский, Квантовый функииональный анализ в бескоординатном изложении, МЦНМО, М., 2009. [11] С. С. Кутателадзе, Основъ функционалъного анализа, изд-во Ин-та математики им. С. П. Соболева, Новосибирск, 1995. [12] G. J. Murphy, $C^{*}$-algebras and operator theory, Academic Press, Boston, MA, 1990. [13] V. Paulsen, Completely Bounded Maps and Operator Algebras, Cambridge Studies Advanced Math., vol. 78, Cambridge University Press, Cambridge, 2002.

Middle East Technical University

Northern Cyprus Campus, Turkey

Поступило в редакцию

e-mail: dosiev@yahoo.com,dosiev@metu.edu.tr 30 ноября 2010 г.

\section{УДК 517.983 .23}

\section{Изометрии с плотными обмотками тора в $C(M)$}

\section{(c) 2012. K. В. Сторожук*}

Пусть $X-$ банахово пространство, $T: X \rightarrow X-$ линейный оператор, такой, что $\left\|T^{n}\right\| \leqslant C<\infty$ для любого $n \in \mathbb{N}$. Положим $X_{0}=\left\{x \in X \mid T^{n} x \rightarrow_{n \rightarrow \infty} 0\right\}$. Оператор $T$ называется асимптотически конечномерным, если codim $X_{0}<\infty$.

Пусть существует компакт $K \subset X$, такой, что орбиты элементов единичного шара $B_{X}$ в том или ином смысле приближаются к $K$. Какие условия на «тот или иной смысл» гарантируют асимптотическую конечномерность оператора $T$ ? Такие же вопросы можно поставить и для однопараметрических полугрупп операторов. Перечислим некоторые условия и результаты.

1. $\lim _{n \rightarrow \infty} \rho\left(T^{n} x, K\right)=0$ для любого $x \in B_{X}$. Тогда $T$ асимптотически конечномерен и даже расщепляем, т. е. $X=X_{0} \oplus L$, где $L$ - конечномерное $T$-инвариантное подпространство. Для марковских полугрупп в $L_{1}$ этот результат доказан в [1], в [2] он установлен для положительных операторов в банаховых решетках, а для произвольного $X$ в [3] и [4].

2. $\limsup _{n \rightarrow \infty} \rho\left(T^{n} x, K\right) \leqslant \eta<1$ для любого $x \in B_{X}$ (т. е. компакт «притягивает, но, быть может, не сильно»). Тогда $T$ асимптотически конечномерен [5].

3. $\liminf _{n \rightarrow \infty} \rho\left(T^{n} x, K\right)=0$ для любого $x \in B_{X}$ (т. е. компакт $K$ «притягивает лишь иногда»). $T$ и в этом случае асимптотически конечномерен [6].

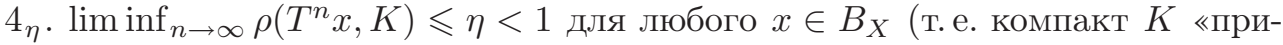
тягивает лишь иногда и не сильно»). В [7, 1.3.33] поставлен следующий вопрос:

*Работа выполнена при поддержке Программы поддержки ведущих научных школ (грант НШ-6613.2010.1). 